\title{
Evolution as Its Own Cause And Effect
}

\author{
George Kampis \\ Department of History and Philosophy of Science \\ Eötvös University, Budapest \\ H-1088 Rakoczi u. 5., Hungary
}

\begin{abstract}
A critique of neo-Darwinism is presented, whith an emphasis on the insufficiency of external selection to explain evolution. Various forms of coevolution are discussed as possible alternatives that offer a biological genesis of evolutionary forces. Problems of self-referentiality are considered with reference to the evolution of closed causal systems. The philosophical perspective of process philosophy is reviewed an its thesis about unfinished identities is amplified in order to present a hypothetical causal mechanism for evolution while avoiding pitfalls of circularity.
\end{abstract}

Key words: Evolution, coevolution, causality, emergence, self-reference, process philosophy

\section{Introduction}

Darwinism is fundamentally incomplete (or, if we are less permissive, fundamentally wrong).

This statement needs some clarification. It would be difficult to deny the existence of differential reproduction, to reject the notion of Malthusian growth, or to challenge the relevance of gradual changes. It is them that bring forth the amazing adaptations we admire when studying evolution. In this sense, Darwinism rests on solid grounds.

In other words, adaptation and selection can certainly be taken as granted. If we are only interested in adaptation and selection, then Darwinism is, in one or another of its modern forms, the very theory we need.

However, I believe that is exactly one half of the story. Evolution theory based on adaptation and selection alone is fundamentally one-sided. It is not just organismic constraints, the Bauplan idea and its relatives, that can modify the adaptationist picture'; the problem has a subtler, and at the same time more central element as well.

Relying on variation and selection in the first place, is like having a theory of classical mechanics based on purely kinetic considerations. Kinetics deals with the speed and the direction of the motion, when the forces have already perfected the shaping of the path. In kinetics, you have trajectories, and you can compute everything about them, but you give no account of the forces, the factors that produce the trajectories. Just as the science of mechanics needed kinematics to explain how forces yield motion, Darwinism in order to become an evolution theory needs a Doppelgänger to explain the nature and origin of selection, responsible for the commencement and sustenance of evolution.

To express the same idea differently: current evolution theory is preoccupied with the "how" of the evolution process (where evolution itself - the very occurrence of the process - is taken as granted); we need now a complementary theory that deals with the "why" part as well, 
explaining the causes of evolution. We shall see that this generativist programme has suggestive consequences, as well as desirable side effects, such as the reduction of omniscience and anticipation built into the theory.

\section{Darwinism as a Scientific Theory}

Do we need an entire new theory? It would be tempting to turn and twist Darwinism, so as to incorporate whatever is missing. Yet I think this is impossible on two grounds. The first is a complex issue, the subject of our paper, whereas the second is a much simpler semantic matter that can be sorted out right now.

The scientific status of evolutionary theory is associated with well-known difficulties. Take the tautology debate of the late seventies ${ }^{2}$. The issue revolved around the circularity of fitness: survival depends on how fit you are, but what determines when are you fit and when are you not? It seems survival is the only proof of this pudding. (Whether there are other ways of pudding-eating we put aside now.) This logic leads to an a posteriori definition of the concept of fitness; we get the tautology "the survivor survives".

Based on the tautology argument, evolution theory was deemed empty by the critics. As a response, defenders pointed out that the theory successfully predicted the existence of survivors and non-survivors, hence it can't be tautologous. Of course, the solution of the paradox is that a theory can retain a certain explanatory value when saying it's a game where there will be winners and losers, even if it is not able to foretell who wins. Yes, but is this what the theory wanted to be about?

At this point we face another, more serious problem. Darwinism as a theory has never been clearly and unambiguously defined. A good part of the otherwise (from the point of view of this paper) unimportant tautology debate was concerned with various formulations of the theory. To put it boldly, nobody knew exactly what Darwinism was. The epigrammatic, general statements offered by J. Maynard Smith or Mary Williams were of little help when it came to the mud-wrestling; it was an open game.

It is widely known that Karl Popper was a passionate opponent of Darwinism exactly on grounds of its notorious flexibility and its openness to endless reinterpretations. He depicted Darwinism as an irrefutable, metaphysical research program. Popper drew a forceful parallel with Marxism, another semantic chameleon of the time, making his pont an easy grasp. Popper later withdrew his critique, but it remains an interesting fact that many, sometimes fundamental amendments and rejoinders have been accommodated in Darwinism without any major change in the general rhetoric or the name of the theory. ${ }^{3}$

Yet we do not have to share Popper's radical taste to recognize that there are limits to obey. It is perhaps reasonable to say that versions of Darwinism (just as of any other theory) possess some core concepts that lends them a common identity. For our purpose, we will say that if this core changes, then we deal with a different theory (research program, paradigm, etc., pick the one you wish). And it is in this sense, not for the sake of being provocative, that the present paper suggests the necessity of a new theory of evolution. ${ }^{4}$ 


\section{The Pan-Competitive View}

Let me start the body of the paper with an hypothetical, straw-man version of the Darwinian theory. This caricature of Darwinism will be claimed to hold that the various evolutionary events (including the "small business" of gene kinetics and the "big business" of speciation) occur as responses to one and the same type of pressure - the one exerted by differential survival. If evolution proceeds by differential survival, and that's all there is, then every species must necessarily compete against every other. The reason why there will be new species is that they do the same job better and better.

Hidden in this seductively simple picture there are several problems, such as those of microversus macro-evolution, or the evolution versus ecology controversy. Lack of clarity nothwithstanding, it is interesting to look at the consequences. The competitive view leads to an essentially "technological" misrepresentation of evolution: if the needs of different species were brought to a common denominator, the latter could consist of nothing but inorganic factors, such as the ones related to efficiency, energy turnover, the mechanics of locomotion, and the like. This picture offers an image of life as an enterprise run in an unfriendly and nonsupportive environment, at a place where you have to fight against cold, the wind, scarcity of food, the lack of water, or its opposite, the abundance of water (in the form of rain, floods, etc.) - a game against Nature that those will win who have a better heat isolation, a bigger ear to hear the prey, or just an umbrella to stay dry.

Needless to say, this bizarre picture has a clear orthogenetic flavor to it, with evolution shown as essentially a functional optimization process. This is the story of how we get from those poor cold-blooded reptiles to the victorious mammals with their controlled body temperature, from gymnosperms to angiosperms, from external to internal fertilization systems, from eggs to wombs, from skin to fur, from $r$ to $K$ strategy, and so on.

Few people would be ready to defend such an oversimplified view, so manifold are its problems. Nevertheless (see footnote 3), this is the skeleton of every Darwinian argument.

We said there are many problems swept under the carpet: one of them is that the story says nothing about why technically inferior solutions don't die out. In reality, they tend to coexist with the "modern" organisms or may (as in the popular case of Gyngko Biloba) spread anew where the technologically more advanced species die out. Also, the story has nothing to say about the origin of such complex adaptations as behavior. Behaviors have little to do with environmental needs whatsoever. They arise "out of nothing", and have a completely internal evolutionary origin. The behavior of the rabbit is a response to that of the eagle, and the behavior of the eagle reflects that of the rabbit. These behaviors (just as many others) can't exist without each other, and they are not necessitated by any global condition or selection pressure.

Furthermore, the technical story is silent about why there is diversity and progression at all why evolution does not come to a halt, to an end state characterized by a single best species, or maybe (if we permit competition to be confined to populations that share common needs) one best species for every lifestyle. Relevant here is the fact that no truly major technical invention took place in the last 70 million years (when we already had those "best" solutions at hand), and this was the perhaps most intensive evolutionary period we know of in life's history. 
I believe the point where the selective-competitive picture is essentially misleading is that it suggests that resources are independently given and therefore the job is to adapt to them. However, resources cannot only be consumed but can also be produced; this is true for ultimately energetic terms (e.g. plants are ecological producers and can also multiply the net energy influx by changing the color of a territory), but also in terms of subtler resources, such as the providing of shelter, or (if you are a carnivore) the formation of large centralized food deposits called big animals. All these goodies depend on entirely biological factors - nothing outside the very process of evolution is responsible for them.

It is other species above all, and not the physical surrounding that constitutes a given species' environment. Evolution takes place in the web of interactions among the species - therefore, natural selection is not a cause but a consequence of the change of this web.

\section{The Tragicomedy of Darwinian Coevolution}

One of the main points of the paper is this: I popose to rediscover and rethink the notion of coevolution. I say "rediscover", since coevolution is not a new idea. Yet with perhaps L. Van Valen's Red Queen Hypothesis (1973) as the only exception, the notion of co-evolution (or mutual evolution) has never been seriously accepted in evolutionary theory. Even the Red Queen is a far too careful (and, therefore, controversial) formulation of a deep fundamental idea. The Red Queen keeps selectionist notions such as fitness and adaptation, and, more importantly, it continues to convey the false impression, amplified by the competitive view, that evolution is a game against something "out there".

What the Red Queen Hypothesis states is that successful adaptation in one species is perceived as the worsening of the environment by others. (Note the term "environment", as if there were any environment independently from evolution; and if my success makes you harm then we are still in competition, aren't we?). As an implication, so continues the argument, other species are challenged to undergo new adaptations to cope with the new conditions. On a mutual basis, this process extends along a progressive braid. You have to enter the rat race in order to stay where you are, or after a few turns you are out.

Expressed properly, I believe this idea has far-reaching consequences. But terms such as "worsening" and the like may not befit situations other than the proverbial rabbit-and-the-fox, where a "better" rabbit means a hungrier predator. How the evolution of species that open various new niches for themselves and for others to interact with can be incorporated in this picture is an open problem, and in fact it is equally unclear how to talk with this theory about anything that does not conform to the logic of arms race where, just as in the Tom and Jerry cartoons, all you will ever get is simply more of the same.

What about the betterment of the environment? Or what about qualitative changes, allowing not for better-or-worse but altogether different, new types of outcomes? ? $^{5}$

The later fate of the Red Queen speaks for itself. It was incorporated as part of Darwinian evolutionary ecology and population dynamics ${ }^{6}$, and today it plays the role of a somewhat perplexed, naive forerunner of Maynard Smith's game theoretic models. ESS models do allow 
for a rational study of the plasticity of evolutionary forces in a multiple actors framework, something the Red Queen has failed to offer.

The game theoretic ESS is a good tool for dealing with genetic variablity in populations, and with behavioral feedback. Still, ESS does not apply to coevolution. You can't consider new genes because they don't have well-defined fitness functions (or payouts), central to the conception. In this interpretation, "coevolution" is an endless, monotonous wandering in one and the same space of solutions (or worse still, an approach to a fixed point). Furthermore, no word is said about the context-dependence of fitness, nor about how fitness is related to the presence or absence of other species, or about how changing aspects of the phenotype enter "fitness" determination ${ }^{7}$.

The situation is much worse if we turn to the standard, more limited interpretations within evolutionary theory of the term "coevolution". Used for such a narrow purpose, coevolution is often just another name for Darwinian evolution. For the entomologist, coevolution may amount to changes in the vegetation, to be included as a parameter on the bug's parallel evolutionary time scale. Whether this sort of biotic simultaneity has to do with the defnition of the basic concepts of evolutionary theory, or with the condition that plants and insects can evolve at all, is none of the entomologist's concern; not his department.

\section{Emergentism and the Open Universe}

Where do the evolutionary forces come from, in the first place? Or where do parameters of the ecological coupling have their origin? Are they present, from time zero on, in an encoded form in the primordial soup? Or is there some causal mechanism that produces them de novo?

Many theorists have articulated the view that evolution must be capable of supplying or generating its own causes en route; the coevolution argument may be the most biological way of coming to a similar position. Other ways are purely philosophical. The emergence of new selective forces as a biological problem will now be considered a subcase of this broader issue.

The emergentists H. Bergson, Ch.L. Morgan, or J.S. Mill have spoken for a dynamic or "creative" view of life that supports causal forms of evolution. Interestingly, the thread begins with Darwin already. He understood that his picture of evolution implies an image of the Universe where things are not created all at once by some omnipotent and omniscient God, but are being created as time goes by. Darwin has realized that no essences, no archetypes, no anticipated forms can exist for such transitory entities of biology. It's a grin of history, that he did not seek open confrontation with Christianity, and he did not amplify these aspects of the theory. ${ }^{8}$

Bergson was concerned with general conceptual problems arising with the metaphors of determinism. He characterized evolution as a productive process where they don't apply. Of the several facets of his criticism of contemporary science, the most pervasive were the ones about time. He spoke of the possibility of motions that do not occupy successions of welldefined states but are open to shape themselves in an interval of time (which he called duration, or durée $)^{9}$. 
The emergentist view received its perhaps clearest and certainly most advanced formulation in Popper's "Open Universe" conception. Popper speaks of an unfinished Universe whose future outcomes are not fully contained in its present - but hints (what he calls propensities) exist. Propensity, or physical possibility, is interpreted by Popper as the occurrence of an individual probabilistic event. Applied to biological evolution, the Popperian view offers the vision of a process with irreducible internal degrees of freedom; in Popper's conception, evolutionary development is seen as an inherent trait of matter, not a response to something else, selection or otherwise.

The Popperian conception is bound to problems and inconsistencies. It denies determinism under all circumstances; with propensities taken seriously, even clocks may not work properly. (That they sometimes don't is a score for Popper, at least within his own system of thinking.) Then, there is the awkward issue of causality versus determinism, where Popper never keeps a clear distinction, obfuscating the question. The line could be continued. Yet it is difficult not to acknowledge that Popper's concept is a truly modern and properly materialistic version of the same theme that bothered many earlier causal evolutionists.

\section{Troubles with the Causal View}

The causal view is not easy to develop, even assuming the truth of one or another of the above constructions. Putting evolution's driving forces inside the living system leads to notorious intellectual puzzles. Let us unveil one of best known specimens of this blend.

The competitive view had an easy job, as its ground terms were well defined at the outset. We have the population, on the one hand, and its environment, on the other. How these terms can be operationalized, and whether they together suffice to explain evolution is another issue, but as far as the logic goes, it is easy to understand on its own. The logic conforms to a scheme familiar from a number of sciences, starting with the example of classical mechanics: there is $\mathbf{A}$ (the environment) that causes $\mathbf{B}$ (genetic change in the population).

At first glance, mutualism may seem to do no harm to this. A makes $\mathbf{B}$ evolve and $\mathbf{B}$ makes $\mathbf{A}$ evolve, no problem as long as we know what we mean by $\mathbf{A}$ and $\mathbf{B}$ - that is, as long as we assume that they are a priori there, having a separate origin, independent from the evolutionary subprocess we are just considering. This was the case of the bug and the plant; presumably, a bug has evolved under bug-making selection forces, and a plant has evolved due to plant-making ones. Now they meet and start evolving together. So be it; all we have to do is to single out those factors that alter the one and alter the other, and we get two nice evolutionary histories that run in parallel.

But already at the ecosystem level things start to bend dangerously backwards, leaning towards themselves. Species of the ecosystem do not have an independent existence any more, not if decoupled from the kinds of evolutionary events they themselves cause when responding to exactly these kinds of events. If we ask what makes the whole ecosystem evolve, and what made it to become what it is now, we are studying a system that has virtually no causal powers outside itself. Studying one part $(\mathbf{A})$ at the expense of another $(\mathbf{B})$ imports the danger that when reversing things ( $\mathbf{B}$ against $\mathbf{A}$ ) we end up in a circularity. 
In a closed system, how is it possible to render a mechanism to its origin? If change in $\mathbf{A}$ causes $\mathbf{B}$ to appear, and change in $\mathbf{B}$ causes $\mathbf{A}$ to appear, then what makes $\mathbf{A}+\mathbf{B}$ appear? Where do we start? The Archimedean point is lost.

Incidentally, it was probably this sort of argument that led Maturana and Varela to adopt a neutralist perspective in the theory of autopoiesis. Autopoiesis is a theory often misunderstood and cited on the wrong occasion. It's a conceptual account of biological existence, with implications for evolution. Maturana and Varela recognized (and doubtlessly they are right) that terms of selection and adaptation do not apply to the systemic level of life. They also realized that it is the systemic level that determines evolution as such (which they express by saying that the evolving system has a certain "autonomy"). At the same time, not being able to associate a governing principle to the closed network of A-to-B-and-B-to-Atype evolutionary interactions, they advanced a neutralist ("drift"-based) theory.

But this thinking is just as erroneous as was neo-Darwinism's optimism that things at large are the same as things in the small. The neutralist or probabilistic perspective (something Popper would be happy to hear about) is biologically empty. It does not conform to the facts. There are just too many adaptations to throw them out.

\section{Circularity Eliminated}

"Self-evoked causes", circularities, or bootstrapping mechanisms like Baron Münchhausen's infamous self-elevating trick always import a suspicion of ill-definedness. Circularity is difficult to digest: in fact, autopoiesis may be the only scientific theory that supports vicious circles, Teufelskreise, and the like.

I am going to argue that the circularity of coevolution is a pseudo-problem. The selfreferential paradox of evolution is an illusory by-product, yielded by a static re-expression of temporally defined dynamic evolutionary forces for which there is no constant "self", apart from a linguistic artifact, to which we could refer.

What I mean to say is this: "A causes $\mathbf{A}$ " does not necessarily imply that $\mathbf{A}$ begets itself (in which case $\mathbf{A}$ would have to be truly circular: empty, undefined or nonexistent). There is another possibility. As a starting point, we should clearly understand that statements of the type "A causes $\mathbf{A}$ " are made possible by the rules of the scientific language. These rules require that the symbol $\mathbf{A}$ stand for the object it stands for: in other words, it assumes that there is such and such an object, conceptualized as an identity translated into propositional content. Then, it is also clear that the problem of self-reference arises at the level of the propositional content and not at that of the causal process.

Paradox can be avoided in more than one ways. Of importance for our subsequent discussion is the possibility that $\boldsymbol{A}$ has never been quite $\boldsymbol{A}$ - that there is no static object underlying evolution. That is, the paradox is bypassed if we think of the objects of evolution as arbitrary labels associated with essentially unknown, complex and flexible things that cannot be exhausted by describing them as $\mathbf{A}$ (or $\mathbf{B}$ or anything else).

This idea is so central to our discussion that I take the liberty to re-formulate it now, first the paradox, then its solution. Take interactions in $\mathbf{A}$, an ecosystem, and take an algebraic model 
of this system. Interactions of $\mathbf{A}$ can never produce $\mathbf{A}$ by a transformation over the connectivity matrix that determines these interactions. The matrix should be given or it will never come about - there is no third possibility. No system has access to its own definition, to its own primitives. Now, how to solve the paradox: what I offer is a rejection of the notion of primitives. The suggestion is that it is different factors in A's material composition, than the ones represented at any time in its mirror image, $\mathbf{A}$, that propel $\mathbf{A}$ towards new elementary evolutionary events. In this way A can cause its own evolution (or, in the long run, its own existence made possible by evolution), by making use of properties and interactions not yet represented in the connectivity matrix, that is, by something in fact not in (what we come to call) A.

\section{Process Philosophy and Relational Biology}

Here we come to the topic of process philosophy: we deal with imperfection of existence and lack of constant identity, as consequences of dormant or previously untested modes of interaction getting activated while others get cancelled.

The content of process philosophy is often identified with Heraclitus' panta rhei or some other idea of irreversibility. We have commonplaces like "everything moves" or its twin "you can never step twice into the same river".

Process philosophy's modern expression is in Whitehead. He draws attention to the fact that identity (of any A together with its propositional content or description) is always a fiction. Whitehead uses trivial examples such as the pendulum that gets rusty, breaks into parts, and stops swinging, to point out the transitory nature of every property - with the permissive remark that science is normally interested in those periods when identity is a good approximation; these are the periods in which pendulums swing and do nothing else.

The escape route offered for many sciences where identity change may be interesting but not important does not apply to evolution. Not only does evolution indeed deal with the longest time intervals possible, and does so almost by definition, but also, coevolutionary scenarios may require a direct use of changing or imperfect identities.

One concept particularly interesting to reconsider is that of relationalism. Relational uses of structures are quite common in evolution; F. Jacob's notion of "evolutionary tinkering" (1982) or Rosen's "principle of function change" (1985) provide examples where they made it to theory. Jacob formulates the thesis that evolution proceeds by re-using old structures in new tasks. In discussing the phenomenon, his concern is with the sub-optimality of evolutionary design due to the use of improper materials, but there is another message as well, that of the non-committed nature of structure. The same organ can function in several ways, depending on the circumstances. Likewise, the same organism (that is, the same body) can show different traits, depending on the context. Rosen's "function change" is a concurrent formulation of the same idea.

In the relational view, there is no such thing as the organism. There are several organisms in one, depending on the interaction modality. If that changes, the same entity presents a different aspect of its fluid phenotype, redefining the evolutionary situation. This suggests we put an emphasis on the flux of spatio-temporal and other factors of interaction instead of any 
kind of material structure, when looking for the determiners of evolutionary properties and evolution itself.

\section{Recursiveness in Evolution}

Applying process thinking, the units of evolution present themselves as relative to the temporary organizations that encompass them, and lend them a character of transitory indentity. Elementary evolutionary change means adaptation to the co-extant "properties", and a simultaneous change of these "properties", without structural transformation.

It is instructive to play with the thought that evolution proceeds by a recursion of such elementary steps. This offers an hypothesis for a causal mechanism that permits evolution, and at the same time explains what sustains it.

That there is a certain recursiveness in evolution was already noticed by several authors, most explicitly by M. Conrad (1979) and O.E. Rössler (1979). One particular instance where recursion is seen at work is the origin of evolutionary capability: life is evolving, but its evolvability should be a result of previous evolution. Sexuality conforms to the same pattern of thought, one of its assumed roles being the maintenance of high genetic variability in populations (i.e. giving food for evolution), with the simultaneous possibility of producing viable offsprings (i.e. reducing the number of selective deaths).

With the incorporation of shifting relational properties, recursiveness can obtain a new dimension in describing coevolution. It may be a basic mechanism for evolutionary change - an idea deserving more discussion than permitted by the limited length of this paper.

\section{Acknowledgment}

This paper was written with the support of a research grant obtained from the Hungarian National Research Fund (OTKA T6428). The first version of the paper was completed during the author's stay at the Department of Logic and Philosophy of Science, University of Basque Country, San Sebastian, Spain. Both sources of support are gratefully acknowledged.

\section{References}

Bergson, H. 1984: Creative Evolution, New York: University Press of America (reprint of 1944 English translation).

Blitz, D. 1992: Emergent Evolution: Qualitative Novelty and the Levels of Reality, New York: Kluwer.

Conrad, M. 1979: Bootstrapping on the Adaptive Landscape, BioSystems 11, 167-182.

Dennett, D. 1995: Darwin's Dangerous Idea, New York: Simon and Schuster.

Goodwin, B. 1994: How the Leopard Changed its Spots: The Evolution of Complexity, New York: Scribners. 
Jacob, F. 1982: The Possible and the Actual, New York: Random House.

Levins, R. 1968: Evolution in Changing Environments, Princeton, NJ: Princeton University Press.

Maturana, H.R. and Varela, F.J. 1980: Autopoiesis and Cognition, Dordrecht: Reidel.

Maynard Smith, J. 1993: The Theory of Evolution, Cambridge: Cambridge University Press (3rd edition of the 1969 original).

Meinhardt, H. 1994: The Algorithmic Beauty of Sea Shells: Shell Patterns as Dynamic Systems, Berlin: Springer.

Maynard Smith, J. 1982: Evolution and the Theory of Games, Cambridge: Cambridge University Press.

Mill, J.S. 1980: Collected Works, London: Routledge.

Morgan, C.L. 1977: Emergent Evolution, New York: AMS Press (2nd reprint edition of the 1923 original).

Nagel, E. 1961: The Structure of Science, New York: Harcourt, Brace and World.

Peters, R.H. 1976: Tautology in Evolution and Ecology, American Naturalist 110, 1-12.

Popper, K.R. 1992: The Open Universe: an Argument for Indeterminism, London: Routledge.

Rosen, R. 1985: Anticipatory Systems, New York: Pergamon.

Rössler, O.E. 1979: Recursive Evolution, BioSystems 11, 193-199.

Stebbins, G.L. 1977: In Defense of Evolution: Tautology or Theory?, American Naturalist 111, 386-390.

Stenseth, N.C. and Maynard Smith, J. 1984: Coevolution in Ecosystems: Red Queen Evolution or Stasis? Evolution 38, 870-880.

Van Valen, L. 1973: A New Evolutionary Law, Evolutionary Theory 1, 1-30.

Varela, F.J. 1979: Principles of Biological Autonomy, New York: North-Holland.

Whitehead, A.N. 1979: Process and Reality, New York: Free Press (corrected edition of the 1926 original).

Wuketits, Franz M. 1987: Charles Darwin, München: Piper. 


\section{NOTES}

${ }^{1}$ Here I refer to the developments of dynamic structuralism. Its representatives put an emphasis on the origin of variations in organisms, a point largely neglected in the now-classical theory of the modern synthesis. For most recent publications, see Goodwin (1994) and Meinhardt (1994).

${ }^{2}$ The debate started with the paper of Peters (1976) and the reply of Stebbins (1977).

${ }^{3}$ It is not the purpose of this paper to use sociology of knowledge or philosophy of science techniques to discuss the cognitive mechanisms underlying this practice, nor is it our aim to look for justifications or counterarguments. A liberal Lakatosian should speak of the normal maturation process of a research program. At the other extreme, an imaginary Vienna Circle neo-positivist would insist that, in lack of a canonical set of sentences uttered in some well-defined, fixed language, Darwinism has never achieved the status of a theory.

${ }^{4}$ For the pedantic, by (neo-) Darwinism hereinafter we mean any explanation that is based on (i) heritable variability and (ii) differential survival; see e.g. Maynard Smith (1993).

${ }^{5}$ The point is that ecological evolution or coevolution is not limited to parallel adaptation but includes something that can be called "innovation", meaning the biological production of new evolutionary forces - in Darwinian terms, new selective forces. This is not restricted to ecology: even a single species is subjected to conditions selected by its own phenotype. For instance, whether its habitat is water, soil, or the air - and so on, up to the minute details - helps determining the physical parameters to which it has to "adapt".

${ }^{6}$ See in particular Stenseth and Maynard Smith (1984).

${ }^{7}$ See Richard Levins (1968).

${ }^{8}$ D. Dennett's most recent book, Darwin's Dangerous Idea (1995) brings a fair review of Darwin's own concepts; another source is Wuketits (1987).

${ }^{9}$ A good summary of Bergson and other radical emergentist ideas is found in Blitz (1992); Nagel (1961) provides famous arguments for why (he thinks) all these ideas are wrong. 